\title{
A novel clinical nomogram for predicting 3-month unfavorable outcomes of stroke patients treated with mechanical thrombectomy
}

\section{Xiao-Guang Zhang}

Tongji University Affilliated Yangpu Hospital: Shanghai Yangpu District Central Hospital Jia-Hui Wang

Tongji University Affilliated Yangpu Hospital: Shanghai Yangpu District Central Hospital

\section{Wen-Hao Yang}

Tongji University Affilliated Yangpu Hospital: Shanghai Yangpu District Central Hospital

Xiao-Qiong Zhu

Tongji University Affilliated Yangpu Hospital: Shanghai Yangpu District Central Hospital Jie Xue

Tongji University Affilliated Yangpu Hospital: Shanghai Yangpu District Central Hospital

\section{Zhi-Zhang Li}

Tongji University Affilliated Yangpu Hospital: Shanghai Yangpu District Central Hospital

\section{Yu-Ming Kong}

Tongji University Affilliated Yangpu Hospital: Shanghai Yangpu District Central Hospital

\section{Liang $\mathrm{Hu}$}

Tongji University Affilliated Yangpu Hospital: Shanghai Yangpu District Central Hospital

\section{Shan-Shan Jiang}

Tongji University Affilliated Yangpu Hospital: Shanghai Yangpu District Central Hospital

\section{Xu-Shen Xu}

Tongji University Affilliated Yangpu Hospital: Shanghai Yangpu District Central Hospital yun-hua yue ( $\square$ Yunhua.Yue@tongji.edu.cn )

Tongji University Affilliated Yangpu Hospital: Shanghai Yangpu District Central Hospital https://orcid.org/0000-0002-5244-1954

\section{Research article}

Keywords: Nomogram, Prediction, Unfavorable outcome, Stroke, Mechanical Thrombectomy

Posted Date: June 14th, 2021

DOI: https://doi.org/10.21203/rs.3.rs-608855/v1 
License: (c) (i) This work is licensed under a Creative Commons Attribution 4.0 International License. Read Full License 


\section{Abstract}

Background: Mechanical thrombectomy (MT) is an effective treatment for large-vessel occlusion in acute ischemic stroke, however, only some revascularized patients have a good prognosis. For stroke patients undergoing MT, predicting the risk of unfavorable outcomes and adjusting the treatment strategies accordingly can greatly improve prognosis. Therefore, we aimed to develop and validate a nomogram that can predict 3-month unfavorable outcomes for individual stroke patient treated with MT.

Methods: We analyzed 238 patients with acute ischemic stroke who underwent MT from January 2018 to October 2020. The primary outcome was a 3-month unfavorable outcome, assessed using the modified Rankin Scale (mRS), 3-6. A nomogram was generated based on a multivariable logistic model. We used the area under the receiver-operating characteristic curve to evaluate the discriminative performance and used the calibration curve and Spiegelhalter's Z-test to assess the calibration performance of the risk prediction model.

Results: After multivariable logistic regression, six variables (gender, bridging therapy, postoperative $\mathrm{mTICl}$, stroke-associated pneumonia, preoperative creatinine and $\mathrm{Na}$ ) remained independent predictors of 3-month unfavorable outcomes in stroke patients treated with $\mathrm{MT}$, thus forming a nomogram. The area under the nomogram curve was 0.848 with good calibration performance $(P=0.946$ for the Spiegelhalter's Z-test).

Conclusions: A novel nomogram consisting of gender, bridging therapy, postoperative mTICl, strokeassociated pneumonia, preoperative creatinine and $\mathrm{Na}$ can predict the 3-month unfavorable outcomes in stroke patients treated with MT.

\section{Background}

Acute ischemic stroke (AIS) is a common cerebrovascular event that can easily cause disability [1]. Mechanical thrombectomy (MT) is essential for AIS with large-vessel occlusion, of which blood perfusion can rarely be restored by intravenous thrombolysis [2]. With the popularization of $M T$, more and more patients have successfully achieved recanalization. However, a successful recanalization of the occluded artery does not always lead to a good outcome (90-day modified Rankin Scale $\geq 2$ ) [3]. Therefore, studies are still needed to predict the potential factors affecting the prognosis of thrombectomy.

Nomogram is a computational tool that can generate numerical probabilities of clinical events based on each patient's personal profile by creating graphical representation of statistical prediction models [4], which has demonstrated significant advantages over traditional staging systems and has been used to predict many diseases, such as pneumonia, myocardial infarction, and cancer [5-7]. In recent years, several prognostic models based on clinical and radiological variables have been used to predict prognosis after thrombectomy for stroke $[3,8,9]$. The patients included in the studies mainly had 
symptoms of acute anterior circulation stroke within $6 \mathrm{~h}$ of onset, representing the largest population of stroke candidates for thrombectomy according to the 2018 guideline for early management of patients with acute ischemic stroke [10]. With the update of the guideline about endovascular thrombectomy, especially the extension of the time windows and the generation of new devices, more patients with indications can benefit from thrombectomy. Therefore, new nomograms for AIS patients undergoing MT are urgently needed.

The present study aimed to develop and validate a nomogram to predict 3-month unfavorable functional outcomes in Chinese AIS patients undergoing MT by using a limited number of easily available variables.

\section{Methods}

\section{Study Population}

We conducted a retrospective study based on data collected from January 2018 to October 2020 from AIS patients who were treated by MT therapy in the Department of Stroke of the Yangpu Hospital, Tongji University School of Medicine. Participants in this study was reviewed and approved by ethics committee of Yangpu Hospital (ethical approval number LL-2018-SCI-004). Informed consent has been obtained. Patients were included in this study if all the following conditions were met: (1) aged $\geq 18$ years; (2) prestroke modified Rankin Scale score (mRS) of 0 to 1 ; (3) ASPECTS of $\geq 6$; (4) with occlusion of the internal carotid artery, anterior cerebral artery, middle cerebral artery, or vertebrobasilar artery confirmed by digital subtraction angiography; (5) undergoing MT treatment within 24 hours of the stroke onset. And patients will receive antithrombotic drugs following the MT treatment. Patients with haemorrhage on baseline or within 24 hours after MT, lost to follow-up without 3-month mRS score, baseline or laboratory data and pre-/post-operative imaging examination were unavailable were excluded.

Demographic characteristics, vascular risk factors, clinical data, radiological images, and laboratory data were all collected. These include age, gender, baseline NIHSS score, baseline ASPECTS score, anesthesia, vascular risk factors such as hypertension, diabetes mellitus, coronary heart disease, atrial fibrillation, smoking and alcoholism, clinical data such as systolic/ diastolic blood pressure, time from onset to puncture, time from puncture to recanalization, bridging therapy, number of retrieval attempts, and strokeassociated pneumonia (SAP), radiological images such as TOAST classification, vascular occlusion site, postoperative modified Thrombolysis in Cerebral Infraction ( $\mathrm{mTICl}$ ) score and symptomatic intracranial hemorrhage $(\mathrm{sICH})$, preoperative laboratory data such as C-reactive protein(CRP), red blood cell distribution width (RDW) , hemoglobin (HGB), glucose, Na concentration, creatinine and B-natriuretic peptide (BNP). The primary outcome was the unfavorable functional consequences at 3 months after stroke, with $\mathrm{mRS}$ between 3 and 6 (i.e., poor prognosis). The 3-month mRS were evaluated by trained physicians with telephone questionnaires or face-to-face interviews.

\section{Statistical analysis}


Continue variables were described as median (interquartile intervals 25-75), and univariate comparisons were performed using Mann-Whitney $U$ where appropriate. Categorical variables were expressed as frequencies and percentages, and differences were assessed by the chi-square test or Fisher's exact test. The glmnet package in $\mathrm{R}$ was used for the least absolute shrinkage and selection operator (LASSO) logistic regression, and significant factors there in were used to enter into the logistic regression model. Using forward stepwise selection with Akaike's information criterion (AIC) as the stopping rule, variables included in the final multivariable logistic regression model were carefully chosen. The AIC value for the final model was minimized with the fewest number of variables. The Variation Inflation Factors (VIF, $<10$ indicating no multicollinearity problem) was used to evaluate the collinearity of combination variables entered into the multivariable logistic regression analysis. The odds ratio (OR) and $95 \%$ confidence interval $(95 \% \mathrm{Cl})$ were calculated for variables significantly associated with the main outcome in the multivariable analysis. Factors remained significant in the multivariable analysis were incorporated in the nomogram.

Based on discrimination and calibration, the discriminative power of the model was assessed. The discrimination of the nomogram model was validated using the receiver operating characteristic (ROC) curve and quantified by the area under the curve (AUC), while the calibration was visually assessed using the calibration plot and the Spiegelhalter's Z-test. A $45^{\circ}$ diagonal line indicates perfect calibration when the predictive value of a well-calibrated model perfectly matches the patient's actual risk. Nomogram construction and calibration was assessed graphically by means of the R package rms.

The statistical analysis was carried out using Stata version 15.0 (Stata Corporation, College Station, TX, USA) statistical software and the statistical software package $R$ version 3.6.0. All tests were two-sided and $\mathrm{P}<0.05$ was considered statistically significant.

\section{Results}

Two hundred and thirty-eight AIS patients were finally enrolled in the entire study (mean age, $73.05 \pm 12.89$ years), 202 patients were treated within 6 hours of symptom onset (Time from onset to groin puncture) and 36 patients treated between 6 and 24 hours after onset of symptom onset. The proportion of patients with poor 3-month pronosis (mRS score 3-6) was 59.7\% (142/238) and 21.4\% (51/238) of patients died during the follow-up period (mRS score $=6$ ). Among them, 35 died of vascular death, 2 died of recurrent ischemic stroke, and 14 died of Non-vascular death. (Table 1)

The clinical, demographic and laboratory characteristics of the patients in the favorable outcome cohorts $(n=96)$ and unfavorable outcome $(n=142)$ cohorts are shown in Table 1. The differences between patients with unfavorable outcome and favorable outcome in age $(p<0.001)$, gender $(p<0.001)$, diabetes mellitus ( $p=0.021)$, smoking $(p=0.032)$, Baseline NIHSS $(p=0.001)$, baseline ASPECTS $(p<0.001)$, bridging therapy $(p=0.006)$, more than one retrieval attempt $(p=0.045)$, vascular occlusion site $(p=0.002)$, postoperative $\mathrm{mTICI}(p=0.003), \operatorname{SAP}(p<0.001), \mathrm{sICH}(p=0.016), \operatorname{CRP}(p=0.018), \mathrm{HGB}(p=0.011), \mathrm{Na}$ $(p=0.018)$, creatinine $(p=0.005)$, and BNP $(p<0.001)$ are significant. 
The LASSO logistic regression algorithm were used to select the most significant predictive factors, which were then used to construct the prediction model. A total of 30 factors shown in Table 1 were used for the LASSO logistic regression, and 11 factors with non-zero coefficients were subsequently selected, with an optimal lambda value of 0.015 (Fig. 1A, 1B). The model ultimately included 11 factors: age, gender, baseline ASPECTS, bridging therapy, vascular occlusion site, postoperative mTICI, SAP, preoperative HGB, $\mathrm{Na}$, creatinine and BNP.

After multivariable logistic regression analysis, gender (OR: $2.65, p=0.008)$, bridging therapy (OR: -3.04 , $p=0.002$ ), postoperative mTICI (OR: $2.13, p=0.033)$, SAP (OR: 4.11, $p<0.001)$, preoperative creatinine (OR: 2.26, $\mathrm{p}=0.024)$ and $\mathrm{Na}(\mathrm{OR}:-3.12, \mathrm{p}=0.002)$ remained independent predictors of 3-month unfavorable outcome (Table 2, Fig. 2). No significant statistical collinearity was observed for any of these six variables. The logistic regression model was generated as: $\log [p(x) / 1-p(x)]=25.19+(1.08$ * gender $)$ $(1.09 *$ bridging therapy $)+(1.49 *$ postoperative $\mathrm{mTICl})+(1.51$ * SAP $)+(0.02 *$ preoperative creatinine $)-$ $(0.19 *$ preoperative $\mathrm{Na})$.

A nomogram was created by assigning a graphical preliminary score ranging from 0 to 100 to each of the six predictors, then summing them to generate a total score, which was then converted into an individual probability of 3-month unfavorable outcome (from $10 \%$ to $90 \%$ ) (Fig. 2). It was predicted that a higher total score of the nomogram was associated with a higher likelihood of unfavorable outcome, while a lower total score was associated with a lower likelihood of an adverse outcome. In this cohort, the nomogram had an area under the curve (AUC) of 0.848 (95\% Cl: 0.80-0.90) (Fig. 3). The p-value for Spiegelhalter's Z-test was 0.946 , which indicated that there was no significant departure from a perfect fit. The Brier was 0.156 , which meant that the calibration curve was almost close to the ideal curve. The calibration plot revealed the adequate fit of the model, predicting the risk of the poor prognosis at 3 months after MT (Fig. 4).

\section{Discussion}

\section{Major findings}

For precise clinical and therapeutic management of AIS patients, early prediction of unfavorable outcome is undoubtedly a valuable perspective. In this study, we developed a nomogram model to predict the unfavorable outcome after MT in AIS with large-vessel occlusion. Our nomogram provides a quantitative prediction of a $10 \%$ to $90 \%$ probability of 3-month unfavorable outcome in Chinese AIS patients undergoing MT, and it exhibits an excellent predictive power with a C-index of 0.848 . Gender, bridging therapy, postoperative $\mathrm{mTICl}$, SAP, preoperative creatinine and $\mathrm{Na}$ are independently associated with poor prognosis at 3-months.

Although $88.24 \%$ of the patients (210/238) benefited from the progressive development of MT intervention materials and prolonged treatment time window to achieve postoperative $\mathrm{mTICl}(\geq 2 \mathrm{~b})$, only $43.81 \%$ of the patients $(92 / 210)$ benefited from the reperfusion. At present, only limited data are available for clinicians to determine the risk of poor prognosis after MT in AIS with large-vessel occlusion. Due to 
the paucity of data, this nomogram was developed specifically for patients undergoing MT within $24 \mathrm{~h}$ from onset to treatment since 2018. This nomogram is based on six basic clinical data, three preoperative data (gender, creatinine and $\mathrm{Na}$ ), one intraoperative data (bridging therapy), and two postoperative data (mTICl, SAP), which demonstrated an excellent discrimination in terms of internal validation.

\section{Nomogram variables interpretation}

In our study, by using a combination of six easily available predictors before and after endovascular procedure, the area under the curve (AUC) of our nomogram could reach 0.848 , which means it may be a novel and reliable graphical computational tool that can provide guidance for AIS patient management after revascularization treatments. Gender differences is also one of the factors affecting the prognosis of stroke. Previous studies reported that females consistently fare worse than males following MT for large vessel ischemic stroke $[11,12]$. One of the possible reasons is that males have larger cerebral arterial diameters than females, which may improve the odds for favorable clinical outcomes [13]. Serum creatinine is widely used as an indicator of renal function, and impaired renal function is also associated with long-term mortality and poor prognosis after stroke [14]. The present study is consistent with previous findings that the worse the renal function, the worse the prognosis. Sodium ions play an important role in maintaining intracellular and extracellular electrolyte gradients, nerve conduction velocity, and muscle excitability [15]. Mild hyponatremia is known to be an independent prognostic factor for mortality in AIS patients [16]. Although the average preoperative Na concentration in the poor prognosis group has not reached hyponatremia, but was lower than that in the good prognosis group, indicating that it could affect the prognosis to some extent. The lower the preoperative $\mathrm{Na}$ concentration, the greater the risk of unfavorable outcomes. For patients with acute ischemic stroke due to anterior circulation large-vessel occlusion, they are eligible for both intravenous alteplase and MT according to most guidelines. Although current DIRECT-MT trial have shown that direct intervention with MT was noninferior to the combination of intravenous thrombolysis followed by MT [17], the trial was still limited to the anterior circulation and time window was 4.5 hours. However, after extending the treatment time window, we found that the benefit of bridging therapy was still greater than that of direct thrombectomy. Successful recanalization after MT is the strongest adjustable predictor of patient prognosis, and higher recanalization rates are associated with functional improvement at 3 months [18]. Successful recanalization of large-vessel occlusions is traditionally defined by $\mathrm{mTICI}$ grades of $2 \mathrm{~b}$ or 3 . Our study also confirmed that the patient prognosis of $\mathrm{mTICl}$ greater than $2 \mathrm{~b}$ was better. Moreover, SAP is another strong predictor of poor outcome in AIS patients undergoing MT. Aspiration caused by dysphagia, reduced consciousness, impaired bulbar reflexes, and hypokinesia are more likely to cause poststroke cumulus pneumonia in AIS patients with MT [19]. For patients with acute stroke, the occurrence of pneumonia has been shown to be associated with poor functional-reelated outcomes and increased risk of death [20]. Therefore, our nomogram covers preoperative, intraoperative and postoperative variables and may be more practical in some respects.

\section{Comparison with prior studies}


Previously, several models have been developed to predict the probability of 3-month mortality in AIS patients receiving MT $[3,8,9]$. Among them, an Italian cohort study developed a nomogram that included NIHSS score, age, pre-stroke mRS score, bridging therapy or direct thrombectomy, grade of recanalization according to the $\mathrm{mTICl}$ grading system, and onset-to-end procedure time [8]. While two Chinese studies identified many demographic and clinical characteristics, including baseline NIHSS score, age, creatinine, blood glucose level, collateral status, and $\mathrm{sICH}[3,9]$. However, due to the inconsistent inclusion and exclusion criteria, the potential predictors finally included in the nomogram in their studies are still quite different. The patients in Cappellari's study had anterior and posterior large-vessel occlusion who underwent thrombectomy within $6 \mathrm{~h}$ of stroke onset [8]. With the update of the guidelines on thrombectomy, the time window for thrombectomy was expanded, and the number of patients requiring endovascular treatment has increased accordingly. The 2018 Guidelines for the Early Management of Acute Ischemic Stroke extended the time window from 6 hours to 24 hours for patients with large-vessel occlusion in the anterior circulation and meeting DAWN or DEFUSE 3 eligibility criteria [10]. So it is currently necessary to update the nomogram to predict the probability of unfavorable outcome in AIS patients who are candidates for thrombectomy. Zhang et al. developed a nomogram that predicted the likelihood of 3-month mortality in patients with anterior circulation stroke who had a successful EVT within 6 hours of symptom onset [3]. Patients with unsuccessful recanalization, non-anterior circulation stroke, exceeding 6 hours of last known normal values were excluded from their study. Based on the fact that unsuccessful recanalization is often accompanied with poor functional outcomes, many patients with poor prognosis may be excluded. While our study assessed AIS patients undergoing MT treatment within 24 hours of the stroke onset with both anterior and posterior circulation infarction.

\section{Nomogram clinical consideration}

We could easily obtain the most significant variables in the nomogram for each patient, indicating that this nomogram is highly practical as a predictive tool. Unlike previous prognostic models [21, 22], our nomogram assigns a probability (from 10 to $90 \%$ ) of unfavorable outcome. For example, our nomogram assigns $>90 \%$ probability of adverse consequence when a male patient ( 0 point), with no bridging therapy ( 18.75 points), postoperative $\mathrm{mTICI} \otimes 2 \mathrm{~b}$ is 22.5 points, SAP is 27.5 points, preoperative creatinine concentration is $160 \mathrm{mmol} / \mathrm{l}$ ( 53.75 points) and $\mathrm{Na}$ concentration is $130 \mathrm{mmol} / \mathrm{l}$ ( 57.5 points), with a total score of 180 points. On the other hand, a male patient ( 0 point), with bridging therapy ( 0 points), postoperative $\mathrm{mTICI} \geq 2 \mathrm{~b}$ ( 0 point), no SAP ( 0 points), preoperative creatinine concentration is $60 \mathrm{mmol} / \mathrm{I}$ (15.5 points) and Na concentration is $144 \mathrm{mmol} / \mathrm{I}$ (12.5 points), with a total score of 28 points, produce a probability of adverse outcome of $<10 \%$.

By converting the total score into a continuum of individual probabilities, our nomogram can more precisely reclassify the risk of 3-month adverse outcome. Thus, the nomogram may better identify different risk predictors in AIS patients undergoing MT than previous prognostic models based on inadequate risk-grouping categorization [23]. 


\section{Limitation}

There are several limitations in this study. First, the data we collected were from a single center, so the sample size was not large enough, which might limit the generalizability of our findings. Further expansion of the sample size is needed to improve the nomogram. Second, biomarkers, such as brain edema and infarct size, were not included in the model because these parameters were not available in the current imaging system. Our future prospective study will include more parameters and determine whether the integration of other biomarkers may help to improve the accuracy of the nomogram. Third, this study is based on a retrospective analysis of prospectively collected data. Despite the good discriminative performance of this model, external validation in completely different patient cohorts is still required.

\section{Conclusions}

A novel nomogram consisting of gender, bridging therapy, postoperative mTICl, SAP, preoperative creatinine and $\mathrm{Na}$ can better predict the 3-month unfavorable outcomes in stroke patients treated with MT.

\section{Abbreviations}

NIHSS, National Institutes of Health Stroke Scale; ASPECTS, Alberta Stroke program early CT score;TOAST, Trial of Org 10172 in Acute Stroke Treatment口ICA, internal carotid artery MCA, middle cerebral artery $\square$ VA, vertebrobasilar artery $\square \mathrm{mTICl}$, modified Thrombolysis in Cerebral Infraction; sICH, symptomatic intracranial hemorrhage; CRP, C-reactive protein; RDW, red blood cell distribution width; HGB, hemoglobin; GLU, glucose; BNP, B-natriuretic peptide and N/A, not applicable.

\section{Declarations}

\section{Acknowledgments}

We thank Chang Shan (Department of Endocrinology and Metabolism, Ren Ji Hospital, School of Medicine, Shanghai Jiao Tong University, Shanghai, China) for her help in revising the manuscript.

\section{Funding}

This work was supported by the Shanghai Sailing Program (20YF1444800), Shanghai Municipal Planning Commission of science and Research Fund (20204Y0123), and the Science and Technology Commission of Shanghai Municipality (18411970100).

\section{Availability of data and materials}

The original contributions presented in the study are included in the article, further inquiries can be directed to the corresponding author. 


\section{Ethics approval and consent to participate}

Participants in this study was reviewed and approved by ethics committee of Yangpu Hospital (LL-2018SCl-004). Written informed consent for participation was required for this study in accordance with the national legislation and the institutional requirements.

\section{Consent for publication}

N/A.

\section{Competing interests}

The authors declare that there is no conflict of interest.

\section{Author details}

${ }^{1}$ Department of Neurology, Yangpu Hospital, Tongji University School of Medicine, Shanghai, China

\section{References}

1 Benjamin EJ, Muntner P, Alonso A, Bittencourt MS, Callaway CW, Carson AP, et al. Heart Disease and Stroke Statistics-2019 Update: A Report From the American Heart Association. Circulation. 2019;139(10):e56-e528. https://doi.org/10.1161/CIR.0000000000000659.

2 Saver JL, Goyal M, Bonafe A, Diener HC, Levy El, Pereira VM, et al. Stent-retriever thrombectomy after intravenous t-PA vs. t-PA alone in stroke. N Engl J Med. 2015;372(24):22852295. https://doi.org/10.1056/NEJMoa1415061.

3 Zhang X, Yuan K, Wang H, Gong P, Jiang T, Xie Y, et al. Nomogram to Predict Mortality of Endovascular Thrombectomy for Ischemic Stroke Despite Successful Recanalization. J Am Heart Assoc. 2020;9(3):e014899. https://doi.org/10.1161/JAHA.119.014899.

4 Xiao L, Jiang Y, Zhang C, Jiang L, Zhou W, Su T, et al. A novel clinical nomogram to predict bilateral hyperaldosteronism in Chinese patients with primary aldosteronism. Clin Endocrinol (Oxf). 2019;90(6):781-788. https://doi.org/10.1111/cen.13962.

$5 \quad$ Kuo TJ, Hsu CL, Liao PH, Huang SJ, Hung YM, Yin CH Nomogram for pneumonia prediction among children and young people with cerebral palsy: A population-based cohort study. PLoS One. 2020;15(7):e0235069. https://doi.org/10.1371/journal.pone.0235069.

6 Tan W, Xie X, Huang Z, Chen L, Tang W, Zhu R, et al. Construction of an immune-related genes nomogram for the preoperative prediction of axillary lymph node metastasis in triple-negative breast cancer. Artif Cells Nanomed Biotechnol. 2020;48(1):288-

297. https://doi.org/10.1080/21691401.2019.1703731. 
7 Zhao E, Xie H, Zhang Y A Nomogram Based on Apelin-12 for the Prediction of Major Adverse Cardiovascular Events after Percutaneous Coronary Intervention among Patients with ST-Segment Elevation Myocardial Infarction. Cardiovasc Ther. 2020;20209416803. https://doi.org/10.1155/2020/9416803.

8 Cappellari M, Mangiafico S, Saia V, Pracucci G, Nappini S, Nencini P, et al. IER-START nomogram for prediction of three-month unfavorable outcome after thrombectomy for stroke. Int J Stroke. 2020;15(4):412-420. https://doi.org/10.1177/1747493019837756.

9 Li X, Zou Y, Hu J, Li XM, Huang CP, Shan YJ, et al. A NAC nomogram to predict the probability of three-month unfavorable outcome in Chinese acute ischemic stroke patients treated with mechanical thrombectomy. Int J Neurosci. 2020;1-7. https://doi.org/10.1080/00207454.2020.1733565.

10 Powers WJ, Rabinstein AA, Ackerson T, Adeoye OM, Bambakidis NC, Becker K, et al. 2018 Guidelines for the Early Management of Patients With Acute Ischemic Stroke: A Guideline for Healthcare Professionals From the American Heart Association/American Stroke Association. Stroke. 2018;49(3):e46-e110. https://doi.org/10.1161/STR.0000000000000158.

11 Silva GS, Lima FO, Camargo EC, Smith WS, Lev MH, Harris GJ, et al. Gender differences in outcomes after ischemic stroke: role of ischemic lesion volume and intracranial large-artery occlusion. Cerebrovasc Dis. 2010;30(5):470-475. https://doi.org/10.1159/000317088.

12 Spaander FH, Zinkstok SM, Baharoglu IM, Gensicke H, Polymeris A, Traenka C, et al. Sex Differences and Functional Outcome After Intravenous Thrombolysis. Stroke. 2017;48(3):699703. https://doi.org/10.1161/STROKEAHA.116.014739.

13 Davison MA, Ouyang B, Keppetipola KM, Chen M Arterial diameter and the gender disparity in stroke thrombectomy outcomes. J Neurointerv Surg. 2018;10(10):949-

952. https://doi.org/10.1136/neurintsurg-2017-013697.

14 Yahalom G, Schwartz R, Schwammenthal Y, Merzeliak O, Toashi M, Orion D, et al. Chronic kidney disease and clinical outcome in patients with acute stroke. Stroke. 2009;40(4):12961303. https://doi.org/10.1161/STROKEAHA.108.520882.

15 Dudev T, Lim C Ion selectivity strategies of sodium channel selectivity filters. Acc Chem Res. 2014;47(12):3580-3587. https://doi.org/10.1021/ar5002878.

16 Gala-Bladzinska A, Czarnota J, Kaczorowski R, Braun M, Gargasz K, Bartosik-Psujek H Mild hyponatremia discovered within the first 24 hours of ischemic stroke is a risk factor for early post stroke mortality. Adv Clin Exp Med. 2019;28(10):1321-1327. https://doi.org/10.17219/acem/103070.

17 Yang P, Zhang Y, Zhang L, Zhang Y, Treurniet KM, Chen W, et al. Endovascular Thrombectomy with or without Intravenous Alteplase in Acute Stroke. N Engl J Med. 2020;382(21):1981- 
1993. https://doi.org/10.1056/NEJMoa2001123.

18 Lin MP, Tsivgoulis G, Alexandrov AV, Chang JJ Factors affecting clinical outcome in large-vessel occlusive ischemic strokes. Int J Stroke. 2015;10(4):479-484. https://doi.org/10.1111/ijs.12406.

19 Smith CJ, Kishore AK, Vail A, Chamorro A, Garau J, Hopkins SJ, et al. Diagnosis of StrokeAssociated Pneumonia: Recommendations From the Pneumonia in Stroke Consensus Group. Stroke. 2015;46(8):2335-2340. https://doi.org/10.1161/STROKEAHA.115.009617.

20 de Jonge JC, Takx RAP, Kauw F, de Jong PA, Dankbaar JW, van der Worp HB Signs of Pulmonary Infection on Admission Chest Computed Tomography Are Associated With Pneumonia or Death in Patients With Acute Stroke. Stroke. 2020;51(6):1690-

1695. https://doi.org/10.1161/STROKEAHA.120.028972.

21 Ntaios G, Faouzi M, Ferrari J, Lang W, Vemmos K, Michel P An integer-based score to predict functional outcome in acute ischemic stroke: the ASTRAL score. Neurology. 2012;78(24):19161922. https://doi.org/10.1212/WNL.0b013e318259e221.

22 Strbian D, Meretoja A, Ahlhelm FJ, Pitkaniemi J, Lyrer P, Kaste M, et al. Predicting outcome of IV thrombolysis-treated ischemic stroke patients: the DRAGON score. Neurology. 2012;78(6):427432. https://doi.org/10.1212/WNL.0b013e318245d2a9.

23 Sun C, Li X, Song B, Chen X, Nyame L, Liu Y, et al. A NADE nomogram to predict the probability of 6-month unfavorable outcome in Chinese patients with ischemic stroke. BMC Neurol. 2019;19(1):274. https://doi.org/10.1186/s12883-019-1464-6.

\section{Tables}

\section{Table 1. Demographics and Clinical Characteristics of study population stratified according to 3-month favorable or unfavorable outcome after acute ischemic stroke in Chinese patients with mechanical thrombectomy.}




\begin{tabular}{|c|c|c|c|}
\hline Variable & $\begin{array}{c}\text { Favorable outcome } \\
\text { (mRS } 0-2)\end{array}$ & $\begin{array}{c}\text { Unfavorable outcome } \\
\text { (mRS 3-6) }\end{array}$ & P Value \\
\hline Patients, n (\%) & 96 & 142 & \\
\hline \multicolumn{4}{|l|}{ Demographic characteristics } \\
\hline Age, y & $68.83 \pm 13.50$ & $75.91 \pm 11.67$ & $\square 0.001$ \\
\hline Female, n (\%) & $32 \square 33.3 \square$ & $82 \square 57.7 \square$ & $\square 0.001$ \\
\hline \multicolumn{4}{|l|}{ Vascular risk factors, n (\%) } \\
\hline Hypertension & $58 \square 60.4 \square$ & $101 \square 71.1 \square$ & 0.085 \\
\hline Diabetes mellitus & 17ロ17.8ロ & $44 \square 31.0 \square$ & 0.021 \\
\hline Atrial fibrillation & $34 \square 35.4 \square$ & $67 \square 47.2 \square$ & 0.072 \\
\hline Coronary heart disease & $16 \square 16.7 \square$ & $39 \square 27.5 \square$ & 0.053 \\
\hline Smoking & $45 \square 46.9 \square$ & $47 \square 33.1 \square$ & 0.032 \\
\hline Alcoholism & 7ロ7.3ロ & $7 \square 5.0 \square$ & 0.447 \\
\hline \multicolumn{4}{|l|}{ Clinical data } \\
\hline Systolic blood pressure, $\mathrm{mm} \mathrm{Hg}$ & $151.94 \pm 20.22$ & $151.70 \pm 26.23$ & 0.941 \\
\hline Diastolic blood pressure, $\mathrm{mm} \mathrm{Hg}$ & $85.34 \pm 15.34$ & $85.65 \pm 14.46$ & 0.874 \\
\hline Baseline NIHSS, score & $13.09 \pm 6.50$ & $16.13 \pm 6.77$ & 0.001 \\
\hline Baseline ASPECTS, score & $8(7-9)$ & $7(6-9)$ & $\square 0.001$ \\
\hline Time from onset to puncture, min & $200 \square 130-270 \square$ & $225 \square 158.75-340 \square$ & 0.146 \\
\hline Time from puncture to recanalization, min & 90ロ55.25-135口 & $85 \square 50-160 \square$ & 0.177 \\
\hline General Anesthesia & $26 \square 27.1 \square$ & $42 \square 29.6 \square$ & 0.676 \\
\hline Bridging therapy & $58 \square 60.4 \square$ & $60 \square 42.3 \square$ & 0.006 \\
\hline More than one Retrieval Attempt & $40 \square 41.7 \square$ & $78 \square 54.9 \square$ & 0.045 \\
\hline \multicolumn{4}{|l|}{ TOAST classification, n (\%) } \\
\hline Large artery atherosclerosis & $54 \square 56.3 \square$ & $70 \square 49.3 \square$ & 0.574 \\
\hline Cardioembolism & $39 \square 40.6 \square$ & $67 \square 47.2 \square$ & \\
\hline Others & $3 \square 3.1 \square$ & $5 \square 3.5 \square$ & \\
\hline \multicolumn{4}{|l|}{ Vascular occlusion site, n (\%) } \\
\hline ICA & $20 \square 20.8 \square$ & $57 \square 40.1 \square$ & 0.002 \\
\hline MCA & $65 \square 67.7 \square$ & $64 \square 45.1 \square$ & \\
\hline VA & $11 \square 11.5 \square$ & $21 \square 14.8 \square$ & \\
\hline Postoperative mTICI $(\geq 2 \mathrm{~b})$ & $92 \square 95.8 \square$ & $118 \square 83.1 \square$ & 0.003 \\
\hline Postoperative pulmonary infection & $27 \square 28.1 \square$ & $100 \square 70.4 \square$ & $\square 0.001$ \\
\hline sICH, n (\%) & $9 \square 9.4 \square$ & $30 \square 21.1 \square$ & 0.016 \\
\hline \multicolumn{4}{|l|}{ Laboratory data } \\
\hline $\mathrm{CRP}(\mathrm{mmol} / \mathrm{l})$ & $5.0 \square 2.46-5 \square$ & $5.12 \square 2.88-13.31 \square$ & 0.018 \\
\hline RDW(\%) & $12.90(12.5-13.4)$ & $13.0(12.6-13.93)$ & 0.332 \\
\hline $\mathrm{HGB}(\mathrm{g} / \mathrm{l})$ & $140.38 \pm 18.38$ & $131.50 \pm 20.61$ & 0.001 \\
\hline GLU(mmol/l) & 6.70ロ5.99-8.04ロ & $8.40 \square 6.70-10.22 \square$ & 0.233 \\
\hline $\mathrm{Na}(\mathrm{mmol} / \mathrm{L})$ & $140.14 \pm 2.69$ & $139.19 \pm 3.39$ & 0.018 \\
\hline Creatinine $(\mu \mathrm{mol} / \mathrm{L})$ & $68.65(60.0-82.0)$ & $72.50(59.93-91.63)$ & 0.005 \\
\hline $\mathrm{BNP}(\mathrm{ng} / \mathrm{l})$ & $112.50(23.25-274)$ & $238.0(98.0-532.75)$ & $\square 0.001$ \\
\hline Death & & & NA \\
\hline Vascular death & 0 & 35 & \\
\hline Recurrent ischemic stroke & 0 & 2 & \\
\hline Non-vascular death & 0 & 14 & \\
\hline
\end{tabular}


NIHSS, National Institutes of Health Stroke Scale; ASPECTS, Alberta Stroke program

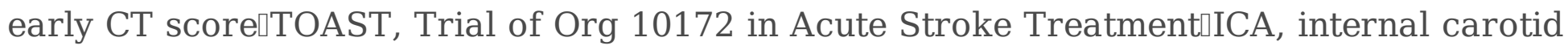

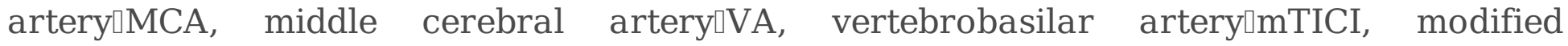
Thrombolysis in Cerebral Infraction; sICH, symptomatic intracranial hemorrhage; CRP, Creactive protein; RDW, red blood cell distribution width; HGB, hemoglobin; GLU, glucose; BNP, B-natriuretic peptide and N/A, not applicable.

Table 2. Multivariable logistic regression model of predictors of unfavorable outcome after acute ischemic stroke in Chinese patients with mechanical thrombectomy.

\begin{tabular}{|l|c|c|l|c|}
\hline & $\beta$ & SE & Odds Ratio (95\% CI) & P value \\
\hline Gender & 1.08 & 0.41 & $2.65(0.28$ to 1.88$)$ & 0.008 \\
\hline Bridging therapy & -1.09 & 0.36 & $-3.04(-1.79$ to -0.39$)$ & 0.002 \\
\hline Postoperative mTICI & 1.49 & 0.70 & $2.13(0.12$ to 2.86$)$ & 0.033 \\
\hline Stroke-associated pneumonia & 1.51 & 0.37 & $4.11(0.79$ to 2.23$)$ & $\square 0.001$ \\
\hline Creatinine & 0.02 & 0.01 & $2.26(0.01$ to -0.03$)$ & 0.024 \\
\hline Na & -0.19 & 0.06 & $-3.12(-0.31$ to -0.07$)$ & 0.002 \\
\hline
\end{tabular}

\section{Figures}


A

$\begin{array}{llllllllllllll}31 & 31 & 31 & 31 & 31 & 29 & 28 & 25 & 23 & 20 & 15 & 7 & 2 & 1\end{array}$

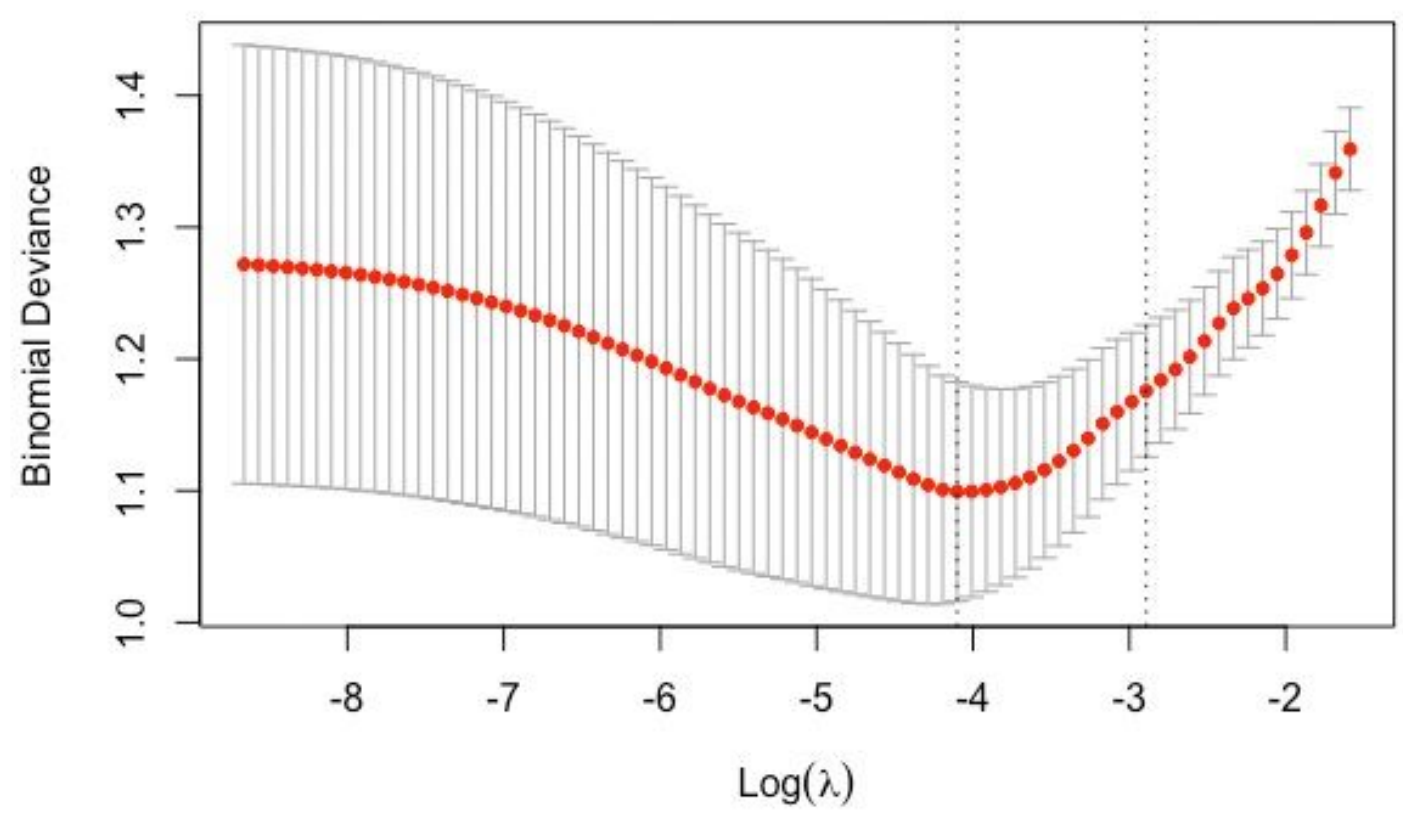

B

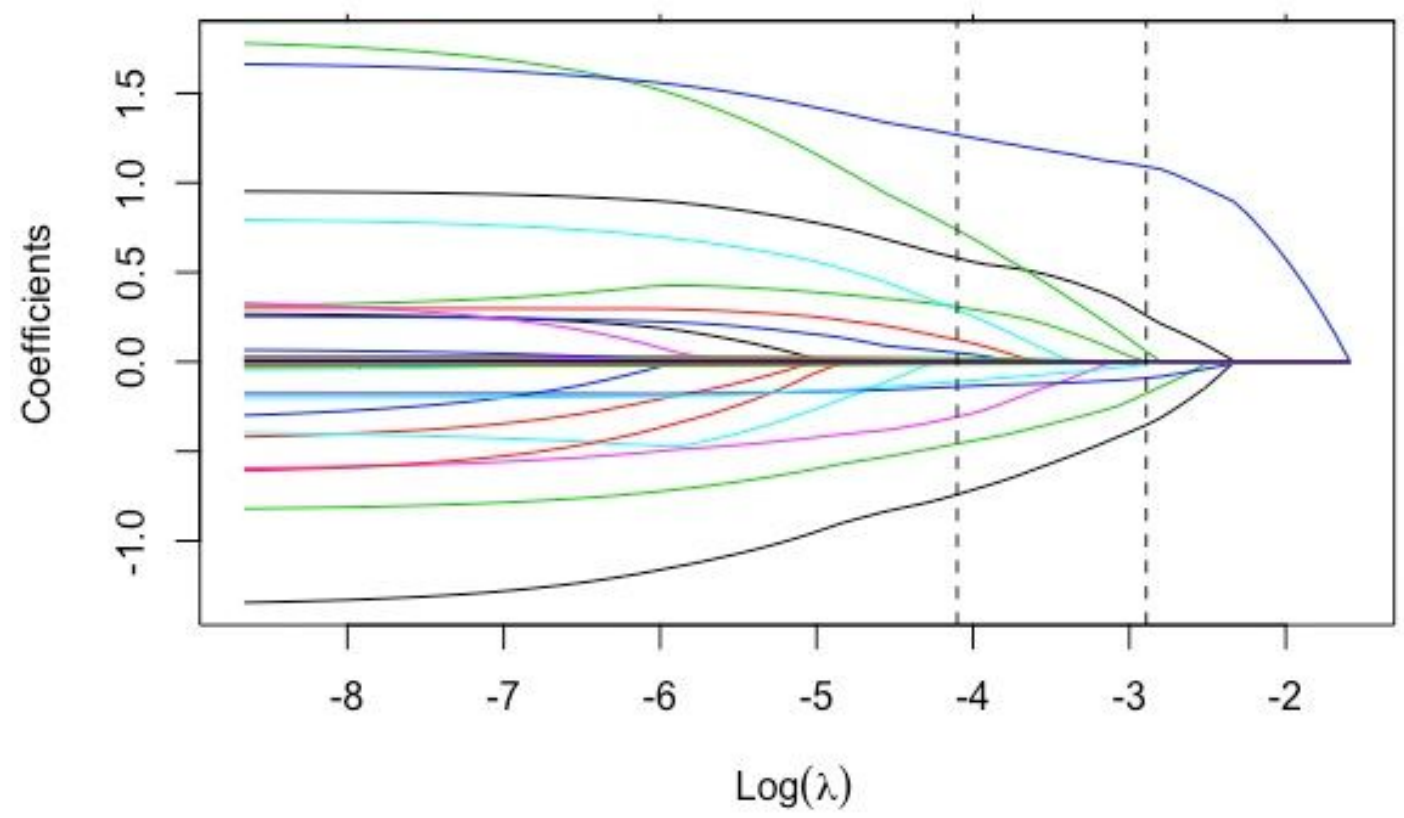

\section{Figure 1}

Factor selection using the least absolute shrinkage and selection operator (LASSO) logistic regression. (A) The LASSO coefficient profiles of the 30 candidate variables. The binomial deviance is plotted versus $\log (\lambda)$. (B) Tuning parameter $(\lambda)$ selection in the LASSO logistic regression performed using 10 -fold cross-validation via the minimum criteria. A coefficient profile plot is produced versus the log $(\lambda)$. The left 
vertical line represents the minimum error, and the right vertical line represents the cross-validated error within 1 standard error of the minimum.

Points

Gender

Bridging therapy

Postoperative mTICI $\geq 2 \mathrm{~b}$

Stroke-associated pneumonia
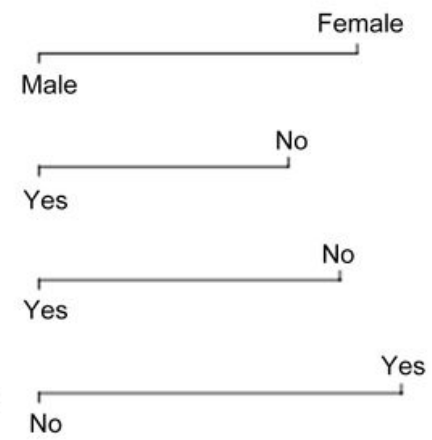

Creatinine

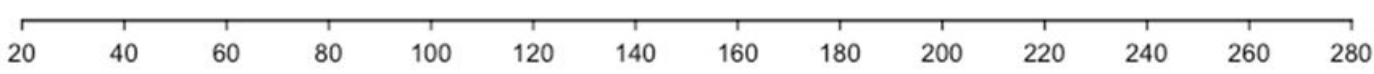

$\mathrm{Na}$

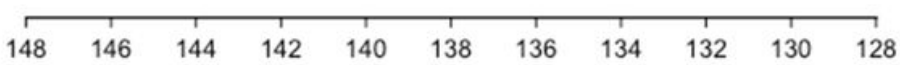

Total points

Linear predictor

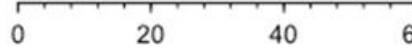

60

80

\begin{tabular}{|c|c|c|c|c|c|c|c|c|c|c|}
\hline-4 & -3 & -2 & -1 & 0 & 1 & 2 & 3 & 4 & 5 & 6 \\
\hline
\end{tabular}

Poor prognosis

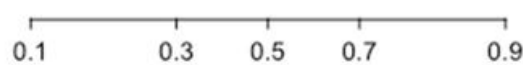

\section{Figure 2}

Nomogram for predicting the probability of 3-month unfavorable outcome in acute ischemic stroke patients undergoing mechanical thrombectomy based on gender, bridging therapy, postoperative $\mathrm{mTICl}$, stroke-associated pneumonia, preoperative creatinine and $\mathrm{Na}$. 


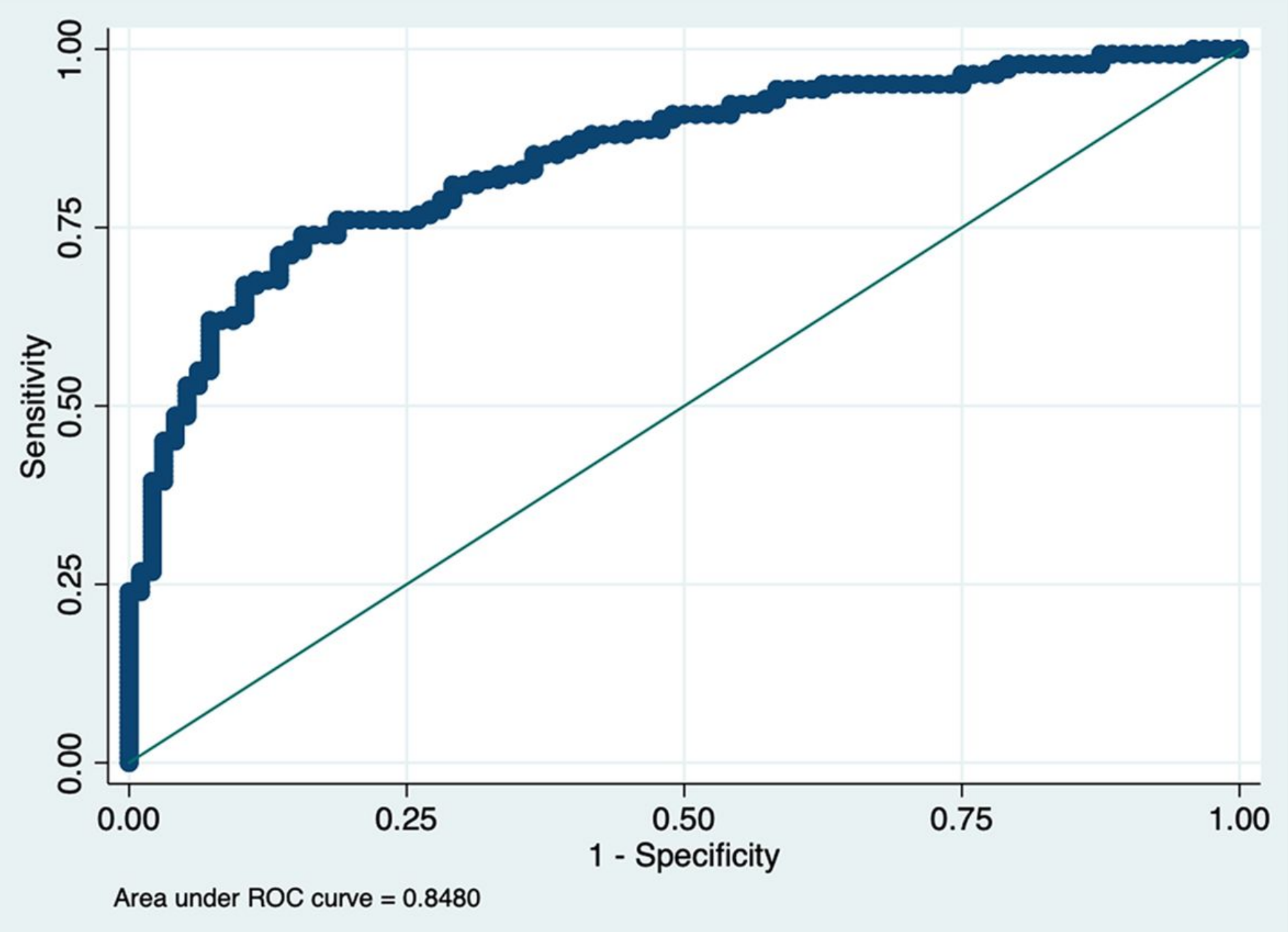

Figure 3

Receiver operating characteristic (ROC) curve of the nomogram for predicting 3-month unfavorable outcomes of stroke patients treated with mechanical thrombectomy. 


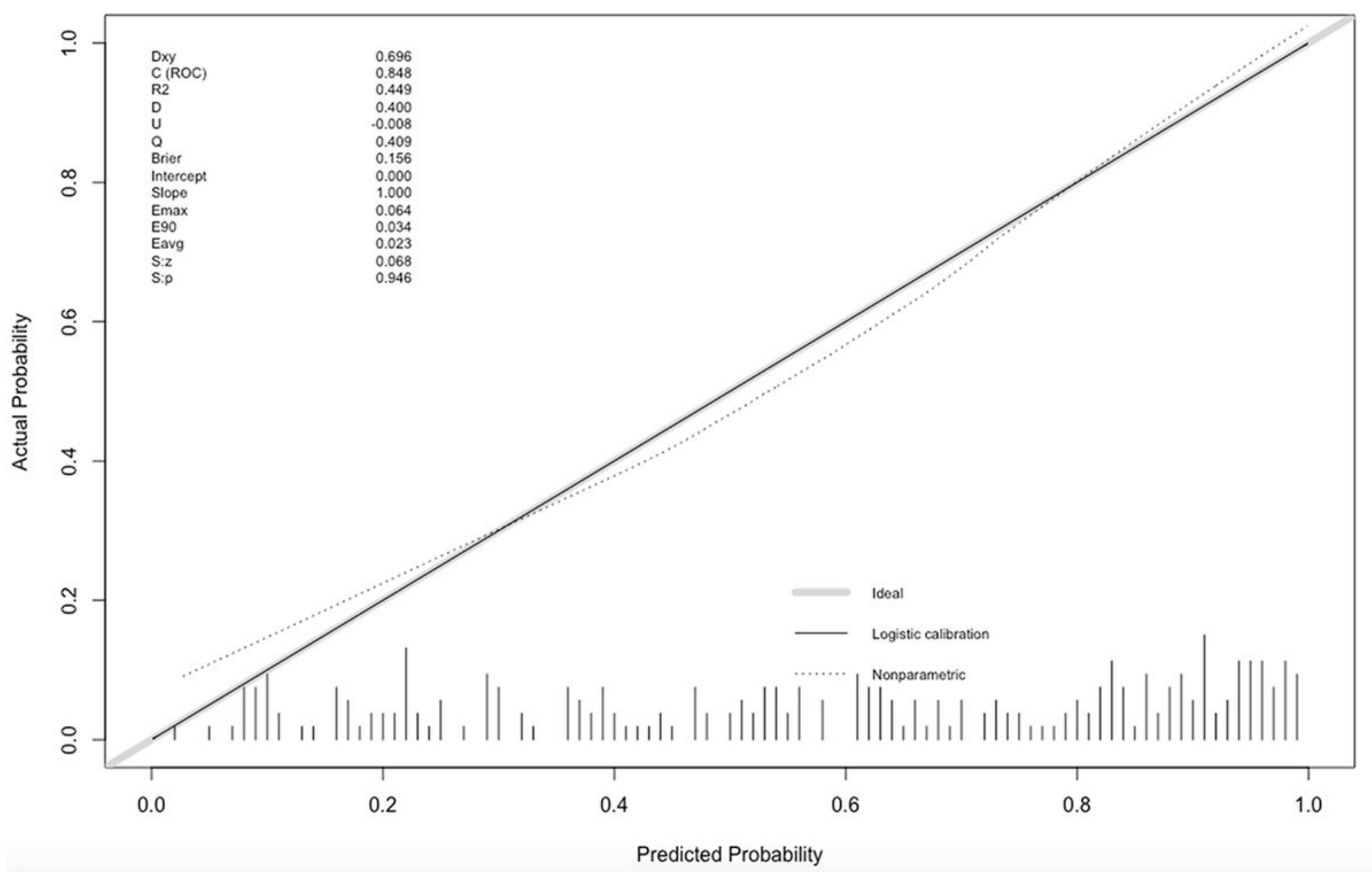

Figure 4

The calibration plot of the nomogram for predicting 3-month unfavorable outcomes of stroke patients treated with mechanical thrombectomy. The x-axis represents the predicted probability of unfavorable outcome calculated using the nomogram. The y-axis represents the actual rate of unfavorable outcome. The dashed line is the reference line where an ideal nomogram would lie. The dotted line is the performance of the nomogram, while the solid line corrects for any deviation of the nomogram. 